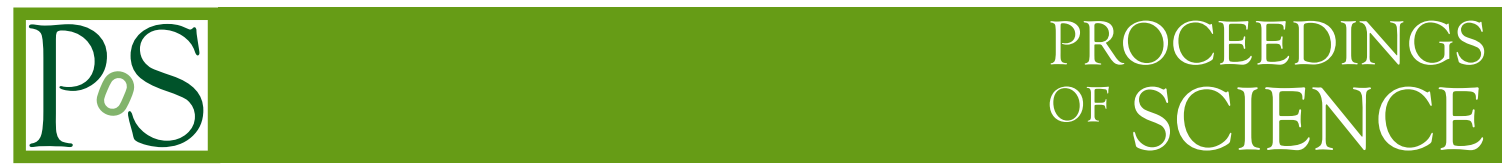

\title{
Results from commissioning and/or prototypes for new small (HL-)LHC experiments
}

\author{
Friedemann Neuhaus ${ }^{a, *}$ for the FASER collaboration \\ ${ }^{a}$ Institute of Physics, Johannes Gutenberg University, Mainz, Germany \\ E-mail: fneuhaus@cern.ch
}

To the surprise of many physicists, no beyond Standard Model particles have been discovered so far at the Large Hadron Collider (LHC). Hence, the search efforts for physics beyond the Standard Model have to be significantly broadened and extended also towards smaller experiments. In this short review, selected results from the commissioning and prototype development of multiple new small-scale experiments at the LHC are presented. The focus is set on the latest results from the FASER experiment, as well as a short overview of the progress of the SND@LHC, MATHUSLA and milliQan experiments.

The Ninth Annual Conference on Large Hadron Collider Physics - LHCP2021

7-12 June 2021

Online

${ }^{*}$ Speaker 


\section{Introduction}

In the last years a number of experiments at the LHC are proposed complementing the physics program set by the four large LHC experiments. While the large experiments focus on the search for heavy particles produced approximately isotropical around the interaction point most new experiments are focussing on either the search for light particles with very weak couplings or weakly charged particles. New light and weakly-interacting particles would be produced parallel to the beam line and could travel long distances through the ground without decaying, i.e. beyond the acceptance of the current LHC detectors. This makes the existing LHC experiments blind to such new particles. Those new particles may be highly collimated, i.e. at angles of $\theta \sim \Lambda_{\mathrm{QCD}} / E$ for new particles produced in pion decays. At energies of $E \sim \mathrm{TeV}$ this would result in a spread of only $\approx 10 \mathrm{~cm}$ at $\approx 480 \mathrm{~m}$ downstream of the Interaction Point (IP). Due to that strong collimation a small detector might be already sufficient to probe for the existence of such new particles making it appealing to design small scale experiments for the far forward region. Those experiments can be realized in short time scales and are relatively cheap compared to the large LHC experiments. The FASER experiment was proposed in November 2018 [1] and installed in March 2021.

\section{FASER Detector and Commissioning}

In this section a summary of the FASER physics case and the FASER detector is given followed by the recent commissioning results of the individual subdetectors. As briefly touched on in the introduction FASER is searching for light and weakly-interacting particles, such as dark photons or Axion-like particles (ALPs), which decay within the FASER detector. In addition, FASER has an interesting neutrino physics program, measuring high-energy neutrino interactions [2]. A detailed discussion of the physics reach is given in [3]. The decay of a dark photon $A^{\prime}$ would be visible through the decay into an electron-positron pair $A^{\prime} \rightarrow e^{+} e^{-}$, while the signature for ALPs is a collimated photon pair.

With those processes in mind the detector must be able to efficiently tag and reject charged particles from the IP, identify oppositely charged particle tracks, reconstruct their origin and measure high-energetic electromagnetic showers [4]. Fig. 1 shows an overview of the full FASER detector with labels for the various subdetectors.
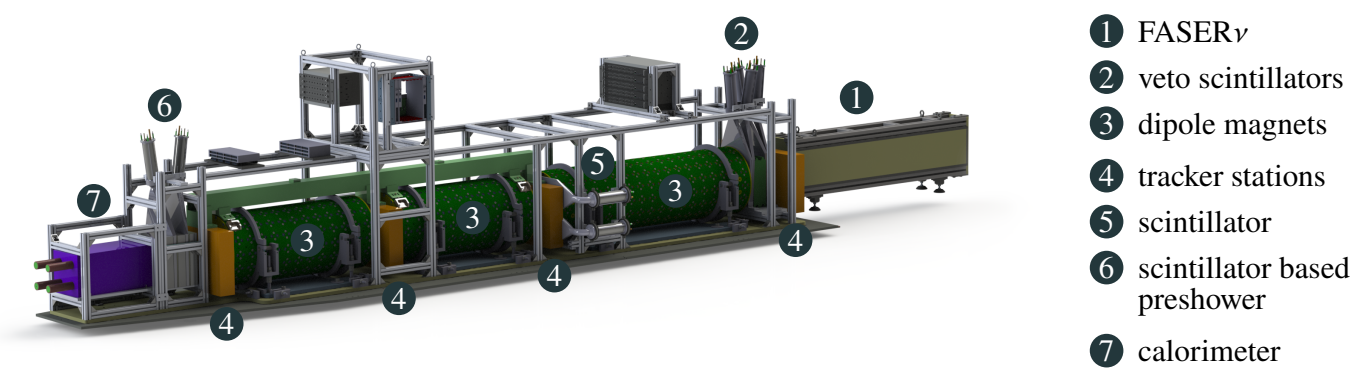

Figure 1: Rendering of the FASER detector with labels for the various subdetectors as seen from the side. The beam line of sight goes centred through the detector from right to left. 


\subsection{Tracker}

The tracker system consists out of three main tracker stations with one station between each magnet pair and one station behind the third magnet. An additional tracker layer will be added as an interface tracker between the FASER $v$ detector and the main FASER detector. Each tracker station is built from three layers with eight silicon detectors each. The silicon detectors used are spare Semi Conductor Tracker (SCT) modules from the ATLAS experiment [5]. Each module has been thoroughly tested before assembling the tracker layers to ensure that it is still within specification after being in storage over multiple years. During on surface commissioning of the individual stations using cosmic muons a resolution of $25 \mu \mathrm{m}$ could be achieved with simple alignment corrections. The three main tracker station have been installed in the cavern and an excellent performance with less than $0.1 \%$ of the strips showing defects.

\subsection{Scintillators}

Another important part of the detector are the three scintillator stations. The first station being the front veto station which needs to veto charged particles with high efficiencies. Using one of the tracker stations and three scintillators the efficiency of the single scintillators is measured with cosmic muons by requiring a straight track through the scintillators. This yielded single layer efficiencies of above $99.995 \%$ for all but one scintillator which is easily within the design specifications.

\subsection{Calorimeter}

The calorimeter consists out of four separate shashlik-style modules with 66 layers of lead absorber and scintillating material $\left(25 X_{0}\right)$ and an active area of $12 \mathrm{~cm} \times 12 \mathrm{~cm}$. The modules are spare outer ecal modules [6] provided by the LHCb experiment along with the corresponding photomultiplier tubes (PMTs) for readout. The PMTs are used in combination with a custom voltage divider base. Both PMTs and modules have been extensively characterized in the lab using LED calibration pulses and cosmic muons. For the in-situ calibration an LED calibration system has been developed and successfully commissioned.

\section{Recent Results and Progress}

\subsection{FASER $v$}

During 2018 a small scale prototype detector for FASER $v$ was placed in an LHC side tunnel, which is exactly on the opposite site from the ATLAS IP compared to the location used for the FASER experiment. It consisted out of $29 \mathrm{~kg}$ lead/emulsion and tungsten/emulsion stacks with around 100 layers.

Due to the limited depth of the prototype detector the track length could not be used to separate between neutrino and neutral hadron vertices. Therefore, a Boosted Decision Tree is used to to classify signal and background events. As a result 6 neutrino event candidates are selected with 3.3 events expected [7]. The full FASER $v$ detector will be significantly larger, i.e. consisting of 770 tungsten layers interleaved with emulsion films resulting in a total mass of $1.1 \mathrm{t}$. In addition to the increased sensitivity due to the large detector, muons can be tagged using the main FASER detector 
allowing for much more precise results on the neutrino interactions. The FASER $v$ detector is being finalized and is scheduled to be installed in time for Run 3.

\subsection{SND@LHC}

A similar experiment to FASER $v$ currently in construction is the Scattering and Neutrino Detector at the LHC experiment (SND@LHC) [8, 9]. It also aims on observing neutrinos produced at an accelerator for the first time and will be located in the side tunnel previously used by the FASER $v$ prototype. SND@LHC is a hybrid detector which is optimized to allow identification of all three neutrino flavours as well as the detection of Feebly Interacting Particles through scattering off atoms in the neutrino target material. The detector construction is progressing well and the experiment is expected to be installed in time for Run 3.

\section{3 milliQan}

The scientific goal of milliQan experiment is the search for milli-charged particles $(Q<$ 0.3e) [10]. The milliQan detector will be located in a tunnel above the CMS experimental cavern and comprised from an array of long scintillating bars pointing towards the CMS IP. During 2018 a small prototype was installed which recorded valuable data used to both validate simulations and to optimize the design for a full scale detector [11]. Two new demonstrator designs are under way for operation during Run 3.

\subsection{MATHUSLA}

The Massive Timing Hodoscope for Ultra-Stable neutraL pArticles (MATHUSLA) is a proposed experiment sensitive to ultra-stable particles with a displaced vertex [12]. With an originally proposed size of $200 \mathrm{~m} \times 200 \mathrm{~m}$ it can not be counted as a small experiment. As a first step, a small prototype has been in place during 2018 located above the ATLAS IP. Using those results an updated Letter of Intent was published in 2020 [13] where the size was reduced to $100 \mathrm{~m} \times 100 \mathrm{~m}$ due to finding of a more suitable location. At the same time the detector technologies were changed reducing complexity and cost. A full Technical Design Report is expected in 2021.

\section{Summary}

In this paper a selection of new experiments is presented illustrating the increased interest in small scale experiments. With FASER and SND@LHC two completely new experiments will be ready for data-taking during the Run-3 of the LHC. Two further experiments, milliQan and MATHUSLA, might be realized in the mid-term future, complementing the physics program of the ATLAS and CMS detectors in highly innovative ways. 


\section{References}

[1] FASER Collaboration, Letter of Intent for FASER: ForwArd Search ExpeRiment at the LHC, 1811.10243.

[2] FASER Collaboration, Technical Proposal: FASERnu, 2001.03073.

[3] FASER Collaboration, FASER's physics reach for long-lived particles, Physical Review D 99 (2019) .

[4] FASER Collaboration, Technical Proposal for FASER: ForwArd Search ExpeRiment at the LHC, 1812.09139.

[5] A. Abdesselam et al., The barrel modules of the ATLAS semiconductor tracker, Nucl. Instrum. Meth. A 568 (2006) 642.

[6] The LHCb Collaboration, The LHCb detector at the LHC, Journal of Instrumentation 3 (2008) S08005.

[7] FASER Collaboration, First neutrino interaction candidates at the LHC, 2105. 06197.

[8] SND Collaboration, Scattering and Neutrino Detector at the LHC, Tech. Rep. CERN-LHCC-2020-013, LHCC-I-037, CERN, Geneva (Aug, 2020).

[9] SND Collaboration, SND@LHC - Scattering and Neutrino Detector at the LHC, Tech. Rep. CERN-LHCC-2021-003, LHCC-P-016, CERN, Geneva (Jan, 2021).

[10] A. Ball, G. Beauregard, J. Brooke, C. Campagnari, M. Carrigan, M. Citron et al., Search for millicharged particles in proton-proton collisions at $\sqrt{s}=13 \mathrm{TeV}$, Phys. Rev. D 102 (2020) 032002.

[11] A. Ball, J. Brooke, C. Campagnari, M. Carrigan, M. Citron, A. De Roeck et al., Sensitivity to millicharged particles in future proton-proton collisions at the LHC with the milliQan detector, Physical Review D 104 (2021).

[12] C. Alpigiani, A. Ball, L. Barak, J. Beacham, Y. Benhammo, T. Cao et al., A Letter of Intent for MATHUSLA: a dedicated displaced vertex detector above ATLAS or CMS, 1811.00927.

[13] MATHUSLA collaboration, An Update to the Letter of Intent for MATHUSLA: Search for Long-Lived Particles at the HL-LHC, 2009. 01693. 